\title{
Characterization of the Role of Nitric Oxide and Its Clinical Applications
}

\author{
Arlene Bradley Levine $^{\mathrm{a}}$ David Punihaole ${ }^{\mathrm{b}}$ T. Barry Levine ${ }^{\mathrm{a}}$ \\ ${ }^{a}$ ABLE Medical Consulting, and ${ }^{b}$ Department of Chemistry, University of Pittsburgh, Pittsburgh, Pa., USA
}

\section{Key Words}

Nitric oxide $\cdot$ Mitochondria $\cdot$ Metabolism $\cdot$ Skeletal/cardiac

myocyte $\cdot$ Insulin resistance

\begin{abstract}
Nitric oxide (NO) has long been known as endothelium-derived relaxing factor. It is a vasodilator, modulating vascular tone, blood pressure and hemodynamics, a role exploited by nitrate donor therapy for angina, heart failure, pulmonary hypertension and erectile dysfunction. In addition, its powerful antioxidant, anti-inflammatory and antithrombotic actions are antiatherogenic with antiatherothrombotic impact. NO signaling modulates skeletal muscle and myocardial contractility and metabolism and is intimately linked with insulin signaling. Vascular and muscle NO signaling coordinate skeletal muscle and myocardial energy demand with supply and are critical for both carbohydrate and fatty acid total-body homeostasis. NO signaling in mitochondria underlies much of NO's metabolic effect, which, at low physiologic levels, links cellular energy demand with mitochondrial energy supply, while beneficially affecting mitochondrial oxidative stress and calcium handling. Mitochondria are also the site for the life-threatening deleterious effects arising from inflammation-related excessive NO levels. NO-deficient states are characterized by cell senescence, oxidative stress, inflammation, endothelial dysfunction, vascular dis-
\end{abstract}

ease, insulin resistance and type 2 diabetes mellitus. NO-enriching therapy would be expected to be of benefit not only for its hemodynamic but also for its metabolic impact. In contrast, strategies are needed to curtail excessive NO in states such as septic shock.

Copyright $\odot 2012$ S. Karger AG, Basel

\section{Introduction}

Nitric oxide (NO) is best known for its actions in the vasculature. In addition, NO plays a key role in cell metabolism and is instrumental in coordinating tissue energy demand with supply [1]. Physiologic NO signaling is pivotal to metabolic and cardiovascular homeostasis. Dysregulation of NO signaling pathways is associated with the pathogenesis of cardiometabolic disorders [2].

\section{History}

NO has a fascinating and colorful scientific history. Based on discoveries, to this day, characterizations of NO run the gamut of being considered a toxic air pollutant, a component of explosives, a proinflammatory and prooxidant mediator or a healthful regulator of vascular and metabolic health.

\section{KARGER}

Fax +4161306 1234

E-Mail karger@karger.ch

www.karger.com
(C) 2012 S. Karger AG, Basel

0008-6312/12/1221-0055\$38.00/0

Accessible online at:

www.karger.com/crd
T. Barry Levine, MD

ABLE Medical Consulting

Pittsburgh, PA 15217 (USA)

E-Mail TBLevine6000@yahoo.com 
NO, a colorless, toxic gas, was first studied by the British theologian and chemist Joseph Priestley in 1772.

In the mid-19th century the French chemist Theophile-Jules Pelouze worked with explosive materials such as guncotton and other nitrosulphates. His student, the Italian chemist Ascanio Sobrero, discovered nitroglycerin (glyceryl trinitrate) in 1847 and noted at that time that exposure to minute quantities of this chemical reproducibly caused severe headaches. Another student of Pelouze, the chemist and engineer Alfred Nobel, combined this very unstable compound with kieselguhr and patented it in 1867 as the more stable commercial explosive dynamite, from which he made his fortune. A serendipitous observation at the time was that factory workers suffering from angina pectoris who were involved in the production of nitroglycerin would find their condition improving during the work week but deteriorating on Sundays upon nitroglycerin withdrawal [3].

Interestingly, Nobel's links with NO go further. In 1879, the British physician William Murrell introduced nitroglycerin as a therapy for angina pectoris. Nobel suffered from angina later in life but refused nitroglycerin as treatment. Nearly 100 years later, in 1977, Ferid Murad found nitroglycerin's beneficial pharmacological effects on vascular smooth muscle to derive from its release of NO [3]. In his last will, Alfred Nobel bequeathed his fortune to the creation of five yearly prizes for individuals who confer the 'greatest benefit on mankind'. One such Nobel Prize was later to be awarded for ' $\mathrm{NO}$ as a signaling molecule in the cardiovascular system', which had been termed 'Molecule of the Year' by Science magazine 1992; the 1998 Nobel Prize in Physiology or Medicine was awarded jointly to Ferid Murad, Robert F. Furchgott, who, with John Zawadski in 1980 recognized the importance of an endothelial-derived relaxing factor in acetylcholine-induced vasodilation, and Louis J. Ignarro, who, with Salvador Moncada, identified endothelial-derived relaxing factor as NO in 1987 [4].

Since the discovery of NO's role in cell signaling, NO has become one of the most researched molecules in recent history. Close to 115,000 scientific articles have been published on NO and its diverse physiologic effects [5].

\section{Nitric Oxide Synthase}

$\mathrm{NO}$ is produced in many tissues by four distinct isoforms of NO synthase (NOS):

(1) neuronal NOS-1 (nNOS),

(2) inducible NOS-2 (iNOS),
(3) endothelial NOS-3 (eNOS) and

(4) mitochondrial NOS (mtNOS) [6].

Whereas iNOS is inducible, eNOS and nNOS are constitutively expressed, continuously elaborating NO. NOS tissue expression is less strict than implied by the nomenclature, and all three isoforms may be constitutive or inducible [6].

NOS consists of a reductase and oxygenase domain. Coupling of the reductase domain of one NOS monomer with the oxygenase domain of its partner is required for proper NO production. The NOS dimer requires nicotinamide adenine dinucleotide phosphate oxidase (NADH/ $\mathrm{NADPH})$, tetrahydrobiopterin $\left(\mathrm{BH}_{4}\right)$ cofactor and oxygen $\left(\mathrm{O}_{2}\right)$ to convert its substrate, $\mathrm{L}$-arginine, to L-citrulline, with the release of the oxidized nitrogen terminal of L-arginine, NO [6]:

\section{(NOS dimer)}

L-arginine $\rightarrow$ L-citrulline $+\mathrm{NO}{ }^{*}$

Molecular $\mathrm{O}_{2}$, rather than $\mathrm{L}$-arginine, becomes the substrate for the uncoupled NOS monomer, generating superoxide $\mathrm{O}_{2} \overline{\text { in lieu of }} \mathrm{NO}$, thus increasing prooxidant stress.

\section{Nitric Oxide Signaling}

\section{NO Bioavailability}

NO is a structurally simple, low-molecular-weight, highly lipophilic free radical. It is extremely reactive, readily forming other nitrogen oxides, which curtails NO bioavailability temporally and spatially:

- NO has a very short half-life;

- NO can travel only limited distances before being oxidized [7].

Nitrite and nitrate $\mathrm{NO}$ reaction products, derivative $\mathrm{S}$ - or $\mathrm{N}$-nitrosoproteins and iron-nitrosyl complexes, are not just inert metabolic waste products. They can be reduced back to release free NO via several pathways [7].

NO bioavailability thus resides not only in the NO radical, but also in NO-containing compounds. These NO products serve as storage pools of bioactive $\mathrm{NO}$ and appear to participate in NO-related processes as they, in contrast to NO, can travel via the circulation to remote tissues [7].

Intracellular Signalosome

Cytosolic oxidants limit NO bioactivity even intracellularly, foiling its diffusion to molecular targets more than approximately $100 \mu \mathrm{m}$ removed from NOS [8]. This 
restricted diffusion, combined with the specific subcellular localizations of NOS, confers specificity and efficiency to NO signaling by confining its actions to protein targets colocalized with NOS within complex multiprotein signalosomes.

\section{NO Signaling}

NO signals via three mechanisms:

(1) Guanylate cyclase activation. By binding to its heme group, NO activates soluble guanylate cyclase, which produces $3^{\prime}-5^{\prime}$-cyclic guanosine monophosphate (cGMP) from guanosine $5^{\prime}$-triphosphate (GTP), the amount generated being proportional to the amount of NO. cGMP activates protein kinase G (cGK) as downstream effector [9]:

(NO:guanylate cyclase)

GTP $\rightarrow$ cGMP $\rightarrow$ activated cGK effector

(2) S-Nitrosylation. NO covalently and reversibly forms S-nitrosothiol groups with reactive cysteine thiols in a wide range of target proteins [10].

(3) Mitogen-activated protein kinases (MAPKs). The intracellular formation of peroxynitrite leads to activation of MAPKs.

Most NO effects are mediated via S-nitrosylation in a cGMP-independent manner [10].

\section{Nitric Oxide Functions}

$\mathrm{NO}$ is a potent signaling molecule, a key determinant of endothelial function, metabolic and vascular health, also affecting the nervous and immune systems. Protective effects occur at pico- to nanomolar NO concentrations. At higher concentrations, $\mathrm{NO}$ and its derivatives become cytotoxic.

\section{Mitochondria}

NO effects on mitochondria have considerable implications for cell physiology and cell death. Mitochondria are primary cellular targets for NO.

mtNOS is linked to mitochondria at several sites of the mitochondrial electron transport chain (ETC), most notably at Complex I (NADH dehydrogenase) [11] and Complex IV (cytochrome c oxidase, CcOX) [12].

mtNOS is highly activated by activation of the ETC and Complex I, which serves as its source of electrons to produce NO. Conversely, inactivation of Complex I terminates normal mtNOS activity [11].

Vascular and Metabolic Effects of Nitric Oxide
Metabolism

mtNOS-derived NO effectively controls mitochondrial respiration, $\mathrm{O}_{2}$ consumption, transmembrane proton gradient and potential and adenosine triphosphate (ATP) synthesis [12].

Acutely, NO reduces mitochondrial oxidative metabolism [13]:

(1) Physiologic NO levels acutely and reversibly bind to and inhibit several ETC complexes, the most sensitive target being Complex IV [12]. The result is a transient NO-induced reduction of mitochondrial respiration with partial mitochondrial membrane depolarization [14]. Since mtNOS derives its electrons from Complex I, there is reciprocal regulation between $\mathrm{mtNOS}$ and the mitochondrial ETC [11].

(2) Very high NO levels, generated upon inflammatory iNOS induction, compete with $\mathrm{O}_{2}$, engendering NO-dependent hypoxia ('nitroxia') [15]. Nitroxia promotes the generation of high levels of reactive oxygen species (ROS)/reactive nitrogen species (RNS) [12]. NO/ RNS can then shut down mitochondrial respiration at multiple sites by irreversibly inhibiting ETC complexes at the expense of ATP production, with cytotoxic effect [16].

Chronically, NO increases cellular oxidative metabolism [13]:

(1) NO-guanylate cyclase signaling increases mitochondrial biogenesis in diverse cell types. NO increases sirtuin-1 expression [17], and, with 5'-AMP-activated protein kinase (AMPK)- $\alpha 1$, synergistically upregulates peroxisome proliferator-activated receptor- $\gamma$ coactivator (PGC)-1 $\alpha$, a master regulator of mitochondriogenesis [13].

ATP formation via mitochondrial oxidative phosphorylation increases in association with the NO/cGMPstimulated increase in mitochondrial content [13] in a variety of tissues.

(2) NO modulates mitochondrial content and totalbody energy balance in response to physiological stimuli, such as exercise or cold exposure, functioning as a unifying molecular switch to trigger the entire mitochondriogenic process [13].

\section{Reactive Oxygen Species}

Mitochondria are the main intracellular source of ROS. Normal oxidative phosphorylation continually produces low ROS/RNS levels, as several ETC redox centers leak electrons to partially reduce $\mathrm{O}_{2}$ to the superoxide anion [18]. Between 0.4 and $4 \%$ of $\mathrm{O}_{2}$ consumed is converted to superoxide.

Cardiology 2012;122:55-68 
The mitochondrial membrane potential is the principal parameter regulating ROS production [18]. Since physiologic NO lowers this potential, NO reduces ROS production [12]. However, disturbed mtNOS function, excessive or deficient $\mathrm{NO}$ and dysregulation of NO signaling pathways increase ROS/RNS production while lowering antioxidant levels [19].

\section{Efficient Mitochondria}

Any increase in energy demand is matched by a coordinated rise in oxidative metabolism, which increases mitochondrial membrane potential and thus ROS generation.

It is paradoxical that $\mathrm{NO} / \mathrm{cGMP}$ signaling decreases oxidative metabolism in any single mitochondrion while increasing cellular mitochondrial function. However, in the process, NO/cGMP renders mitochondria 'efficient', with an organized ETC that generates sufficient ATP, while lowering oxygen consumption, mitochondrial potential and ROS production.

The result is of major benefit. Exercise training increases energy demand but also stimulates NO, since NO couples demand with cellular and total-body energy generation [20]. Instead of the expected ROS increase, oxidative stress is reduced due to 'efficient' mitochondria, forestalling ROS-induced cellular aging by protecting the integrity of mitochondria, telomeres or the endoplasmic reticulum.

\section{Mitochondrial Calcium}

Mitochondrial energy homeostasis responds to changes in mitochondrial $\mathrm{Ca}^{2+}$. Key mitochondrial enzymes, as in the tricarboxylic acid cycle, are upregulated by higher intramitochondrial $\mathrm{Ca}^{2+}$, enhancing the provision of reducing equivalents to the ETC and increasing mitochondrial potential and ATP generation [16].

Cytoplasmic $\mathrm{Ca}^{2+}$ signals correspond to higher energy demand from secretory, contractile or other work. Thus, a primary function for mitochondrial $\mathrm{Ca}^{2+}$ uptake appears to be the $\mathrm{Ca}^{2+}$-dependent coordination of mitochondrial energy production with cellular energy consumption.

$\mathrm{Ca}^{2+}$ uptake into mitochondria is partly driven by the mitochondrial membrane potential. Excessive mitochondrial $\mathrm{Ca}^{2+}$ accumulation is implicated in disease.

Lowering the mitochondrial potential limits mitochondrial $\mathrm{Ca}^{2+}$. Such conditions not only lower mitochondrial metabolic activity but also protect against deleterious $\mathrm{Ca}^{2+}$ overload [16].

$\mathrm{NO} / \mathrm{cGMP}$ reduces the mitochondrial potential, thereby decreasing mitochondrial $\mathrm{Ca}^{2+}[14]$. In fact, $\mathrm{NO}$ pro- vides negative feedback on mitochondrial $\mathrm{Ca}^{2+}$ uptake: whereas higher mitochondrial $\mathrm{Ca}^{2+}$ activates mtNOS, increasing NO inhibits respiration, lowering the mitochondrial potential and further limiting $\mathrm{Ca}^{2+}$ uptake [14].

\section{Cell Protection}

Ischemic preconditioning provides powerful cardioprotection against myocardial ischemia-reperfusion injury. Physiologic NO levels are involved in cytoprotective effects of early and late preconditioning. Not only eNOS-, but also exogenous nitrate-donor-derived NO can effect endothelial and myocardial cytoprotection [21].

$\mathrm{NO/cGMP}$ may protect against mitochondrial permeability transition and apoptosis induced by manifold insults. Through its interaction with ETC components, such as CcOX, NO affects low-level ROS generation and other mitochondrial defense mechanisms, thereby triggering adaptive cell survival signaling $[15,21]$.

\section{Cell Death}

High NO concentrations are cytotoxic:

(1) Excessive NO and RNS, such as peroxynitrite, may cause tyrosine nitration of mitochondrial components and play a key role in apoptosis [19].

(2) NO-derived ROS/RNS signaling, mitochondrial permeability transition or DNA damage may activate mitochondrial pathways to apoptosis or necrosis.

(3) The irreversible inhibition of mitochondrial respiration at multiple sites by excessive NO can inhibit apoptosis and induce necrosis via energy depletion. The ensuing profound mitochondrial failure contributes to the insidious, progressive and fatal end-organ failure of sepsis, associated with signs of accelerated and refractory anaerobic metabolism [22].

\section{Skeletal Muscle}

NO signaling in skeletal muscle is implicated in the control of multiple functions, including

- muscle metabolism,

- excitation-contraction coupling and contractility,

- immune function,

- cell growth and

- neurotransmission.

Metabolically active skeletal muscle is the most abundant tissue, constituting approximately $40 \%$ of normal-weight body mass, rendering it a critical factor in total-body metabolism [23]. Skeletal muscle NOS thus plays a pivotal role in total-body glucose and lipid homeostasis. 
Glucose

Higher skeletal muscle NOS expression and activity improve insulin action via $\mathrm{NO} / \mathrm{cGMP} / \mathrm{cGK}$ signaling [23].

Insulin sensitivity is enhanced

- indirectly as NO increases

- skeletal muscle microvascular perfusion, delivering nutrients and insulin to target tissues [23],

- antioxidant and anti-inflammatory actions,

- the synthesis of insulin-sensitizing adiponectin;

- directly as NO/cGK blocks the inhibitory interaction of the small GTPase Rho/Rho kinase with insulin receptor substrate (IRS)-1 [24].

In contrast, excessive proinflammatory iNOS/NO induction impairs myocyte insulin sensitivity via prooxidant pathways.

Glucose uptake and myocyte intracellular energy stores are also stimulated by $\mathrm{NO} / \mathrm{cGMP} / \mathrm{cGK}$ signaling and NOS-derived ROS via mechanisms that are distinct from, but additive to, contraction-, insulin-, AMPK- or p38 MAPK-dependent glucose uptake pathways [23, 25].

NO stimulates glucose oxidation in skeletal and cardiac muscle, liver and adipose tissue via cGMP-dependent mechanisms.

\section{Fatty Acids}

Increased physiologic NO/cGMP signaling enhances fatty acid catabolism [13]. It accelerates adipocyte lipolysis while stimulating fatty acid oxidation in skeletal and cardiac muscle via AMPK activation and PGC- $1 \alpha$ expression [26].

Oxygen Consumption

NO reduces myocyte energy demand [23] by

- reducing contractility. $\mathrm{NO}$ reduces myofilament $\mathrm{Ca}^{2+}$ sensitivity through nitrosation of target proteins, depressing submaximal and isometric skeletal muscle force, shortening contraction velocity and accelerating relaxation;

- downregulating metabolism. NO lowers glycolysis. It reduces mitochondrial respiration, the breakdown of creatine phosphate and the transfer of high-energy phosphates.

\section{Contractile Dysfunction}

Cardiac pump failure is a life-threatening response to severe inflammation in myocarditis, heart transplant rejection, sepsis or trauma. Excessive myocardial iNOS/ $\mathrm{NO} / \mathrm{cGMP} / \mathrm{cGK}$ induction has a profound negative inotropic effect [27] as it

Vascular and Metabolic Effects of Nitric Oxide
- inhibits aerobic enzymes, including $\mathrm{CcOX}$,

- depresses cAMP levels, thereby reducing $\mathrm{Ca}^{2+}$ influx through L-type $\mathrm{Ca}^{2+}$ channels and

- phosphorylates troponin I, lowering myofilament $\mathrm{Ca}^{2+}$ sensitivity.

\section{Myocyte Loss}

Human diseases, ranging from heart failure to cancer, induce skeletal muscle catabolism via proinflammatory induction of excessive iNOS/NO, which impairs myocyte differentiation and is associated with myocyte apoptosis. There is also a significant association between iNOS abundance, cardiomyocyte apoptosis and cardiomyopathy.

\section{Vasculature}

Vascular NO is produced by endothelial cells.

Vasodilation

$\mathrm{NO}$ is the most potent endogenous vasodilator, predominantly of conduit vessels rather than the microvasculature.

$\mathrm{NO} / \mathrm{cGMP} / \mathrm{cGK}$ signaling accomplishes vasodilation through

- the autocrine increase of $\mathrm{NO}$ and $\mathrm{BH}_{4}$ within the endothelium [9],

- the paracrine relaxation of subjacent vascular smooth muscle cells (VSMCs) by

(1) lowering cytoplasmic $\mathrm{Ca}^{2+}$ concentrations and

(2) reducing myofibrillar $\mathrm{Ca}^{2+}$ sensitivity $[9,24]$.

NO mediates flow-mediated vasodilation and opposes vasoconstrictor effects. It counteracts vascular stiffness and lowers blood pressure. NO is a critical modulator of blood flow, vascular tone and blood pressure [28].

Vascular Repair and Angiogenesis

The endothelium is continuously exposed to mechanical, chemical or ischemic insults. At the site of injuries, bone marrow-derived endothelial stem and progenitor cells (EPCs) participate in repair processes, normalizing endothelial function. NO protects the functional ability of EPCs to participate in vascular repair and angiogenesis [29].

\section{Inhibition of Platelet Activation}

NO inhibits platelet activation, aggregation and adhesion to the endothelium via cGMP-dependent [9] and -independent mechanisms. 


\section{Oxidative Stress}

Physiologic NO levels reduce oxidative stress. NO inhibits superoxide production by inactivating NADH/ NADPH oxidase. NO increases the endogenous antioxidant potential by inducing endothelial superoxide dismutase (SOD), extracellular SOD in VSMCs, myocardial SOD, mitochondrial S-nitrosoglutathione synthesis [11] and thioredoxin activity [30], thus thwarting oxidative NO inactivation. NO inhibits low-density lipoprotein (LDL) oxidation.

In contrast, induction of high levels of NO/iNOS is highly prooxidant. $\mathrm{NO}$, reacting with superoxide, generates the oxidant anion peroxynitrite $\left(\mathrm{ONOO}^{-}\right)$:

$$
\mathrm{NO}+\mathrm{O}_{2}^{\overline{2}} \rightarrow \mathrm{ONOO}^{-}
$$

Peroxynitrite engenders lipid peroxidation and nitrosation of amino acid residues, disrupting cell membranes, cell signaling and cell survival [30]. Peroxynitrite also has proinflammatory effects.

\section{Anti-Inflammatory and Antiatherogenic Activities}

Physiologic NO levels are anti-inflammatory. By preventing proinflammatory cytokine activation, NO protects blood vessels from endogenous injury, interfering with early and later stages of conduit vessel atherogenesis [28]. NO

- delays endothelial cell senescence and senescence-related proinflammatory signaling,

- reduces endothelial cell apoptosis,

- inhibits the transcription of nuclear factor- $\kappa B$,

- inhibits redox-sensitive, cytokine-induced vascular cell adhesion molecule-1, intracellular adhesion molecule- 1 and monocyte chemoattractant protein-1, preventing leukocyte adhesion to the endothelium,

- decreases endothelial permeability, reducing the influx of oxidized lipoproteins into the vascular wall,

- interferes with leukocyte migration into the vascular wall by decreasing the expression of factors, including the surface adhesion molecules CD11/CD18 and P-selectin,

- powerfully inhibits inflammatory cell activation and monocyte activity,

- blocks VSMC migration,

- thwarts VSMC proliferation,

- inhibits the synthesis and secretion of extracellular matrix proteinases, which degrade extracellular matrix proteins,

- increases the expression of tissue inhibitor of matrix metalloproteinases,
- inhibits transforming growth factor- $\beta /$ Smad-regulated gene transactivation $[31,32]$.

\section{Causes of Reduced NO}

Diminished NO bioactivity reflects an imbalance between its synthesis and degradation. There may also be impaired VSMC responsivity to NO. The pathophysiological mechanisms involved are multifactorial and differ with diverse etiologies.

\section{Decreased eNOS Expression}

In advanced coronary heart disease (CHD), eNOS expression may be reduced due to reduced transcription of, and/or cytokine- or lipid-induced instabilities of, eNOS mRNA [33], impairing NO release.

\section{Decreased eNOS Activity}

Gene Polymorphism

eNOS gene polymorphisms, such as Glu298 $\rightarrow$ Asp, may lower enzyme activity and basal NO production. This substitution is responsible for a significant frequency of endothelial dysfunction, hypertension, vasospastic angina, CHD and cardiovascular mortality [34].

\section{Arginase}

L-Arginine deficiency is rare but may occur with increased L-arginine metabolism. Arginases hydrolyze Larginine, thus lowering eNOS activity by competing for L-arginine. Arginase plays an important role in the pathogenesis of reduced $\mathrm{NO}$ and endothelial dysfunction with proinflammatory conditions, aging and diseases like diabetes mellitus (DM) [35].

Asymmetric Dimethylarginine

Endogenously produced competitive inhibitors of Larginine, such as asymmetric dimethylarginine (ADMA) and $\mathrm{N}$-monomethylarginine, can create a relative deficiency of the natural substrate for eNOS, thus curtailing NO production. These endogenous inhibitors of NOS activity may be responsible for the endothelial dysfunction of individuals with CHD risk factors and/or CHD $[9,33]$.

\section{Decreased Cofactor Availability}

$\mathrm{BH}_{4}$ is very susceptible to oxidation. $\mathrm{BH}_{4}$ deficiency uncouples NOS, thus lowering NO output, increasing ROS production and engendering endothelial dysfunction [9]. 
Decreased NO Half-Life

Once released, NO half-life is reduced with oxidative stress, causing endothelial dysfunction. Superoxide scavenges $\mathrm{NO}$ to form peroxynitrite and other prooxidants [33]. Myeloperoxidase catalytically consumes NO.

\section{Mixed Effects}

Oxidative stress also inhibits NO production by impairing eNOS expression and activity. Oxidized LDL inactivates NO directly and decreases NO release [31].

Inflammation

Inflammation reduces NO bioavailability [32].

Tumor Necrosis Factor- $\alpha$. Proinflammatory tumor necrosis factor (TNF)- $\alpha$ downregulates eNOS expression. It inhibits shear stress-mediated NO production. TNF- $\alpha$ and interleukin- $1 \beta$ increase iNOS expression with cross-activation of protein kinase $\mathrm{A}$, downregulating $\mathrm{cGK}$ expression. TNF- $\alpha$ increases oxidative stress by increasing NADH/ NADPH expression, undermining NO bioavailability [36].

Angiotensin II. Proinflammatory upregulation of angiotensin II potently increases prooxidant stress through stimulation of vascular/leukocyte $\mathrm{NADH} / \mathrm{NADPH}$. It also decreases $\mathrm{NO} / \mathrm{cGMP} / \mathrm{cGK}$ action by stimulating phosphodiesterase (PDE), which increases cGMP hydrolysis, decreasing cGMP/cGK levels and action [9].

Endothelin-1. High inflammatory endothelin (ET)-1 levels lower NO production via ET A receptor action. ET-1 also induces endothelial NADH/NADPH, increasing oxidant stress at the expense of NO bioavailability.

Rho/Rho Kinase. Inflammatory cytokines and vasoconstrictors signal via RhoA/Rho kinase. RhoA/Rho kinase suppress both eNOS activity and expression, causing rapid and prolonged reduction of NO production [24].

Glucocorticoids. Stress activation of cortisol significantly decreases eNOS expression in a dose-dependent manner and reduces agonist-induced NO release. Glucocorticoids also impair $\mathrm{BH}_{4}$ synthesis.

Insulin Resistance

Under normal physiological circumstances, insulin stimulates NO production in endothelial cells.

Insulin resistance elicits disturbances of intracellular signal transduction that lower NO bioavailability:

impaired phosphatidylinositol 3-kinase-Akt pathway

$\downarrow$
decreased eNOS activation
$\downarrow$
decreased NO bioavailability.

Table 1. Factors that reduce NO bioavailability

\begin{tabular}{|c|c|}
\hline $\begin{array}{l}\text { Hypertension } \\
\text { Dyslipidemia } \\
\text { Free fatty acids } \\
\text { Hyperuricemia } \\
\text { Angiotensin II } \\
\text { NADH/NADPH activation } \\
\text { Proinflammatory cytokines } \\
\text { Glucose intolerance }\end{array}$ & decreased NO production/activity \\
\hline
\end{tabular}

For example, a mouse model for knockout of the insulin receptor or IRS-1, in addition to the expected metabolic defects, engenders impaired endothelium-dependent vascular relaxation and hypertension. IRS-1/2-null mice develop atherosclerosis. A mutation of IRS-1 (Arg792) creates abnormal vasoreactivity, lower eNOS expression and a higher incidence of CHD [37].

As insulin resistance worsens and progresses to the metabolic syndrome, components of the syndrome secondarily worsen NO bioavailability [38], as shown in table 1.

\section{Shear Stress}

The physiologically most important determinants of NO generation and local blood flow regulation are fluid shear stress and pulsatile stretch. Laminar shear stress, the tangential, mechanical dragging force exerted by fluid flow over the endothelial surface, is one of the most important physiologic stimuli for NO release from the vascular endothelium [39]. Laminar shear stress elicits multiple synergistic mechanisms to enhance NO

- acutely, by activating eNOS and NO release to dilate the blood vessel, reducing shear stress toward normal, and

- chronically, by enhancing eNOS expression.

Endothelial function is preserved with steady laminar flow at approximately $12 \mathrm{dyn} / \mathrm{cm}^{2}$ on the endothelium or oscillatory flow that remains unidirectional.

NO production and endothelial cell function are disturbed by

- stasis,

- low net flow,

- low shear stress at approximately $0.4 \mathrm{dyn} / \mathrm{cm}^{2}$,

- turbulence,

- local shear gradients,

- rapidly changing flow and

- oscillatory flow with flow reversal, 
which promote inflammatory activation, oxidative stress and atherogenesis [40]. Additionally, decreased arterial wall compliance and higher pulse pressure adversely modulate flow signal effects on the vessel wall.

Although the entire vasculature is exposed to identical risk factors, early atherosclerosis develops in regions of bifurcations, branches and inner curvatures, where blood flow characteristics are complex and associated with disturbed and/or low shear stress conditions. The presence of systemic risk factors further modifies regional endothelial phenotype and focal susceptibility to atherosclerosis [40].

Inactivity. Physical inactivity is associated with low net blood flow, low shear stress and stasis. Inactivity begets NO deficiency, insulin resistance and its inflammatory and catabolic pathways. Prolonged rest reduces eNOS expression and impairs endothelium-dependent vasodilation [41]. Even short-term sedentary living impairs endothelial function in the absence of other cardiovascular risk factors.

Cell Senescence. Senescent cells are proinflammatory, prooxidant and insulin resistant. With advancing age, cell aging becomes a predominant cause of impaired NO bioavailability. Aging-related cardiometabolic dysfunction is greatly compounded by the toll of inactivity.

\section{Cardiovascular Risk Factors}

All traditional, as well as new, cardiovascular risk markers, including

- physical inactivity,

- weight gain and obesity,

- postprandial state following a high-fat, carbohydraterich meal,

- oxidative stress,

- infection/inflammation, elevated C-reactive protein and proinflammatory cytokines,

- mental stress,

- insulin resistance, hyperglycemia, metabolic syndrome, DM,

- dyslipidemia,

- hyperhomocysteinemia,

- hypertension,

- a family history of premature atherosclerotic disease,

- established CHD, peripheral/cerebrovascular disease and heart failure,

- increasing age and

- cigarette consumption,

activate diverse pathways that impair $\mathrm{NO}$ bioavailability [42].

\section{Manifestations of Reduced NO}

Reduced physiologic NO signaling and increased superoxide formation by dysfunctional NOS are pathogenic and contribute to the clinical course of cardiometabolic disease [43].

\section{Abnormal Vascular Function}

Impaired NO bioavailability is a key feature of vascular dysfunction [42].

\section{Endothelial Dysfunction}

Decreased NO bioavailability attenuates NO-dependent flow-induced vasodilation in conduit and resistance vessels, defining endothelial dysfunction [42].

Impaired NO bioavailability and endothelial dysfunction are a necessary first stage in the transition from normal vascular function to vasoconstriction, inflammation, atherogenesis, overt atherosclerosis and thrombosis. An abnormal brachial or coronary vasodilatory response of the endothelium to increased blood flow is a major independent predictor of atheromatous disease progression and cardiovascular event rates in individuals at risk for CHD [42].

\section{Vasoconstriction}

Decreased NO bioavailability engenders loss of other endogenous vasodilators, including endothelium-derived hyperpolarizing factor and prostacyclin, with an increase in vasoconstrictors [42].

Flow-induced vasodilation is attenuated with aging due to impaired NO bioavailability and augmented vasoconstriction, one of the earliest markers of vascular dysfunction. In patients with hypercholesterolemia and coronary atherosclerosis, coronary and systemic arteries may constrict during exercise, reflecting both the loss of vasodilatory capacity and the increased responsiveness to vasoconstrictors, such as norepinephrine and ET-1 [39].

Mice deficient for the eNOS gene have hypertension [44]. Diminished NO signaling contributes to the clinical course of systemic and pulmonary hypertension. The elaboration of ET-1, vasoconstrictor prostanoids and angiotensin II engenders VSMC contraction, salt and water retention and mitogenic effects, promoting the development of hypertension [42].

\section{Inflammation}

Impaired NO bioavailability promotes inflammation. Vascular inflammation is increased in eNOS(-/-) mice 
[45]. Upregulation and activation of nuclear factor $-\kappa B$ and activator protein- 1 initiate the release of inflammatory cytokines, such as TNF- $\alpha$ and interleukin-1. As T lymphocytes migrate into the vascular intima, they produce further cyto- and chemotactic factors, as well as adhesion molecules, to recruit VSMCs and monocytes, initiating atherogenesis [42].

\section{Atherogenesis}

NO bioavailability is inversely related to the progression of atheromatous vascular disease [43].

The dysfunctional endothelium engenders an endothelial phenotype that promotes vascular remodeling. Chronically deficient NO activity contributes to medial thickening, myointimal hyperplasia and increased conduit vessel stiffness [42].

In the absence of $\mathrm{NO}$, the continued elaboration of proinflammatory factors promotes further $\mathrm{T}$ cell and monocyte adhesion, foam cell formation, extracellular matrix digestion, VSMC migration and proliferation, initiating and accelerating atherosclerotic plaque formation [33].

\section{Atherothrombosis}

Deficient vascular NO activity engenders the loss of antithrombotic factors. There is decreased expression of cell surface-based thrombomodulin, attenuating anticoagulation. Reduced NO and prostacyclin allow for enhanced platelet activation and aggregation [42].

Deficient NO increases prothrombotic factors. Production of the fibrinolytic antagonist plasminogen activator inhibitor-1 is enhanced [42]. Dysfunctional endothelial cells produce the powerful coagulant tissue factor. These developments elevate the risk for atherothrombosis, particularly in the later disease stages, culminating in plaque rupture, thrombus formation and acute ischemic syndromes [33, 43].

The cardiovascular event rate among patients with severe coronary endothelial dysfunction is $14 \%$ over 2 years of follow-up and approximately $20 \%$ over 8 years, versus $<5 \%$ for individuals with normal endothelial function [13].

\section{Abnormal Muscle Metabolism}

Abnormal NOS/NO function curtails muscle mitochondrial content, oxidative phosphorylation and aerobic exercise capacity [39].

Mutant eNOS-null(-/-) mice have reduced mitochondriogenesis and metabolic rate but accelerated weight gain relative to wild types. Their brain, kidney, liver, heart and gastrocnemius muscle display markedly reduced mitochondrial content and size with significantly lower oxygen consumption and ATP content. The markedly lower beta-oxidation in subsarcolemmal mitochondria of such mice is associated with a significant increase in ectopic intramyocellular lipid content relative to controls, a risk for the development of insulin resistance [2].

Dysfunctional NO signaling contributes to impaired exercise capacity in insulin resistance: while dysfunctional NOS reduces NO-mediated capillary recruitment, nutritive skeletal blood flow and glucose uptake during exercise, insulin resistance diminishes muscle perfusion, glucose uptake and glycogen restoration during recovery, lowering functional exercise capacity, the anaerobic threshold and peak oxygen consumption. It also lowers meal-induced thermogenesis, engendering a tendency to gain weight when compared to individuals with normal metabolism [23].

\section{Insulin Resistance}

Dysfunctional NOS links vascular and metabolic pathways and cardiovascular and metabolic disease [44]. Deficient NO is implicated in the pathogenesis of insulin resistance. Insulin-resistant states have reduced expression of endothelial and skeletal muscle NOS with reduced activity [23].

Loss of NOS expression at endothelial and skeletal muscle sites engenders insulin resistance, hyperlipidemia and impaired insulin-stimulated glucose uptake [44]. eNOS(-/-) mice are insulin resistant [45] with increased triglycerides and free fatty acid levels. Diminished NO availability, caused by ADMA administration to wild-type mice, impairs insulin sensitivity within hours.

Decreased NO bioavailability and endothelial dysfunction develop at an early stage, prior to carbohydrate intolerance, and may constitute an early link not only to insulin resistance and hyperglycemia but also to the cardiometabolic pathophysiologic sequelae of the metabolic syndrome.

\section{NO-Directed Therapy}

Physiologic NO levels play a key role in metabolic and cardiovascular homeostasis. Well-preserved NO signaling predicts good mitochondrial function, exercise tolerance, endothelial function, insulin sensitivity and the absence of cardiometabolic disease. 
In the setting of deficient systemic $\mathrm{NO}$, exogenous $\mathrm{NO}$ delivery is an attractive option for improving cardiometabolic health. A number of lifestyle changes and medical interventions that enhance NO bioavailability also improve insulin sensitivity and cardiometabolic risk and are highly effective treatments for cardiovascular disease [46].

NO has a feature in common with the Goldilocks story; although too little is not good, too much is devastating. NO bioavailability has to be just right.

Diet

Systemic NO levels are generated not only through endogenous NOS. Diet is a safe and inexpensive mode of increasing $\mathrm{NO}$ bioavailability.

Calorie restriction induces eNOS expression and cGMP generation. This is accompanied by the enhanced expression of Sirt1 and mitochondriogenesis [17, 47].

Black, green, oolong or white tea polyphenols, including epigallocatechin gallate, promote catalytic eNOS activity. Tea consumption may reverse endothelial dysfunction and beneficially affect weight control and insulin sensitivity.

Red wine polyphenols, including resveratrol, quercetin and gallic acid, upregulate eNOS expression and NO production, which, in turn, significantly enhance the function of circulating EPCs, with beneficial cardiometabolic effect.

Flavonol-rich cocoa consumption increases circulating NO with vasculoprotective, insulin-sensitizing impact. In general, fruit- and vegetable-derived flavonoids increase NO bioactivity.

Green leafy vegetable consumption raises levels of vasculoprotective nitroso-compounds. Vegetables, the dominant dietary nitrate source, increase tissue and plasma levels of bioactive nitrogen oxides, improving blood pressure and reversing a prediabetic phenotype.

\section{Exercise}

The cardiometabolic toll of inactivity is reversible, even in the aged [1]. Since skeletal muscle is the most abundant tissue, barring excessive adipose expansion, adaptations in the skeletal, cardiac muscle and vascular NO systems account for many of the significant cardiometabolic benefits of exercise training.

Exercise training augments endothelial and skeletal NOS expression and activation, comprehensively matching higher energy supply with increased demand. Exercise-induced repetitive increases in laminar shear blood flow, as well as in acetylcholine diffusion from neuro- muscular junctions, enhance NO signaling, endothelial function, capillary density and nutritive blood flow, while upregulating oxidative metabolism, metabolic rate, insulin sensitivity and glucose uptake and reducing incident DM. Exercise attenuates norepinephrine-mediated abnormal coronary vasoconstriction even in $\mathrm{CHD}$ patients and raises parasympathetic input to the heart.

Exercise-mediated increases in $\mathrm{NO}$ also enhance telomeric integrity and mitochondrial efficiency, reducing oxidative stress and proinflammatory signaling.

Exercise effects are greatest in vascular beds subtending working muscle groups. However, due to changes in heart rate, pulse pressure, blood viscosity and flow, exercise-related vascular shear stress enhances NO bioactivity systemically, also in hypertensive, $\mathrm{CHD}$ and heart failure patients [39].

\section{Intensity}

Moderate exercise (25-75\% maximal oxygen consumption) enhances plasma nitrite/nitrate levels, SOD activity and endothelial function. Mild exercise has no impact. However, strenuous physical training $(>75 \%$ maximal oxygen consumption) increases oxidative stress, undermining the benefits of exercise.

\section{Time}

NO training adaptations reduce resting blood pressure after as little as 4 weeks [23]:

- Short-term adaptations focus largely on NO-mediated vasodilation.

- Longer-term (weeks to years) adaptations entail NOmediated vascular remodeling, such as increases in vessel diameter.

\section{Pharmacologic Approaches to Increasing NO}

NO inhalation is used in limited applications, such as in pulmonary hypertension and in neonates.

More commonly, NO donor drugs, including organic nitrates and sodium nitroprusside, are employed to ensure the stable delivery of NO for its vascular/hemodynamic effects in a range of applications, including angina, heart failure, pulmonary hypertension, hypertensive crises or erectile dysfunction. Their long-term use has been limited by the development of nitrate tolerance and toxicity issues.

NO's high reactivity has allowed the development of novel NO donors with varying modes or rates of NO release. However, despite significant research efforts, no novel NO donors have met approval for clinical use [8]. 
A number of drugs do increase NO bioavailability or its downstream signaling.

Angiotensin-Converting Enzyme Inhibition

Traditionally, angiotensin-converting enzyme (ACE) inhibitors elicit their effects by inhibiting the conversion of angiotensin I to angiotensin II, thus diminishing the local, vascular and systemic adverse effects of the latter.

ACE inhibitors also improve endothelial-derived NO production, endothelial function, VSMC relaxation and vascular compliance with beneficial hemodynamic impact. ACE inhibitors

- reduce oxidant inactivation of NO,

- increase bradykinin levels, enhancing NO production,

- upregulate eNOS expression and activity,

- reduce plasma levels of ADMA and

- increase plasma levels of nitrate and nitrite.

Although they may not reverse atherosclerosis, ACE inhibitors clinically stabilize and slow the progression of atherogenesis, reducing clinical cardiovascular events and stroke. ACE inhibitors improve insulin sensitivity and glucose metabolism and lower the rates of new-onset type $2 \mathrm{DM}$ in high-risk individuals by up to $14 \%$ [48].

\section{Angiotensin II Receptor Blockade}

Although the mechanisms of action of ACE inhibitors and angiotensin receptor blockers (ARBs) differ, their clinical effects are similar, and ARBs share many of the beneficial effects of ACE inhibitors.

ARBs improve endothelial function, reverse endothelial dysfunction and have vasculoprotective effects likely due to antagonism of the vascular renin-angiotensin-aldosterone system with reduced $\mathrm{AT}_{1}$ receptor-mediated effects, lowered oxidative stress, anti-inflammatory modulation, decreased plasma levels of ADMA and increased NO bioavailability.

The concomitant elevation in plasma and tissue angiotensin II levels with ARB therapy may provide vascular protection also via unopposed $\mathrm{AT}_{2}$ receptor stimulation, the effects of which may be mediated in part via $\mathrm{NO}$ and bradykinin generation. These beneficial vascular effects also occur in patients with hypertension and CHD and are independent of a blood pressure-lowering effect.

ARBs improve insulin sensitivity and glucose tolerance and reduce the new onset of type 2 DM [49].

\section{$\beta$-Adrenergic Blockade}

Third-generation, vasodilating $\beta$-blockers have beneficial metabolic and vasculoprotective effects. Different drugs have distinct effects:

Vascular and Metabolic Effects of Nitric Oxide
- Nebivolol, a $\beta$-blocker highly selective for $\beta 1$-adrenergic receptors, causes vasodilatation by augmenting eNOS activity, NO release and bioavailability and endothelial function. It reduces NADH/NADPH activity and oxidative stress, increases adiponectin levels and lessens systemic insulin resistance [50].

- Celiprolol, a selective $\beta 1$-blocker, stimulates eNOS expression and has $\beta 2$-stimulating properties. It induces vasodilation and may improve insulin action.

- Carvedilol is an $\alpha 1-, \beta 1$ - and $\beta 2$-adrenergic receptor blocker which augments eNOS activity. It has ancillary vasodilatory capacity, anti-ischemic and antioxidant effects. Carvedilol improves endothelial function and insulin sensitivity and may lower the incidence of type 2 DM.

\section{PDE-5 Inhibitors}

PDE-5 inhibitors, which include sildenafil, vardenafil and tadalafil, may be of potential benefit for vascular and metabolic health [51].

PDE-5 is abundant in most vascular beds, particularly in VSMCs of the corpus cavernosum and the pulmonary artery. PDE-5 mediates the breakdown of cGMP.

By increasing intracellular cGMP, PDE-5 inhibition exerts a potent vasodilatory effect. PDE-5 inhibitors may also improve eNOS expression and activity and release endogenous vasodilators, such as adenosine and bradykinin, that may, in turn, trigger NO release. PDE-5 inhibitors thus effectively enhance penile blood flow and reduce pulmonary vascular resistance, and are used in the therapy of erectile dysfunction and pulmonary hypertension, respectively.

PDE-5 inhibitors protect endothelial function in general, in chronic heart failure and CHD patients. They may have antioxidant effects and improve insulin sensitivity and pancreatic $\beta$-cell function.

The 3-Hydroxy-3-Methylglutaryl-Coenzyme A

Reductase Inhibitors

The 3-hydroxy-3-methylglutaryl-coenzyme A (HMG$\mathrm{CoA}$ ) reductase inhibitors, also termed statins, are the only lipid-lowering drugs conclusively shown to save lives. In a systematic review of 97 randomized, controlled trials of lipid-lowering interventions, statin use was the most favorable pharmacologic lipid-lowering strategy that reduced risks for overall and cardiac mortality.

As their name implies, statins inhibit HMG-CoA reductase, which catalyzes the rate-limiting step in hepatic cholesterol synthesis, the conversion of HMG-CoA into 
Table 2. The parallel evolution of vascular and metabolic disease

$\left.\begin{array}{l|l}\left.\begin{array}{l}\text { Chronic infection } \\
\text { Inflammatory disease } \\
\begin{array}{l}\text { Mental stress } \\
\text { Irritants }\end{array} \\
\begin{array}{l}\text { Insomnia } \\
\text { Sleep apnea } \\
\text { Age }\end{array}\end{array}\right\} \begin{array}{l}\text { mitochondrial dysfunction } \\
\text { telomere erosion } \\
\text { senescence } \\
\text { inflammation } \\
\text { oxidative stress }\end{array} & \begin{array}{l}\text { dysfunctional NO signaling } \\
\text { insulin resistance }\end{array}\end{array}\right\}$\begin{tabular}{l}
$\begin{array}{l}\text { hypertension } \\
\text { cardiomyopathy } \\
\text { coronary and peripheral vascular disease } \\
\text { metabolic syndrome } \\
\text { type 2 DM }\end{array}$ \\
\hline
\end{tabular}

mevalonate. By competitively binding to hepatic HMG$\mathrm{CoA}$ reductase, statins interfere with cholesterol and isoprenoid synthesis.

Statin effects on dyslipidemia do not account for all of the observed improvements in vascular risk reduction. eNOS plays an important role in mediating their beneficial pleiotropic effects. However, statins may vary in their efficacy to enhance NO release:

- in endothelial cells, statins increase eNOS mRNA half-life without changing eNOS gene transcription;

- statins may increase eNOS activity by enhancing eNOS phosphorylation by Akt;

- statins may potentiate eNOS activation by promoting agonist-induced eNOS association with the chaperone heat shock protein 90 ;

- statins prevent eNOS downregulation by oxidized LDL, and

- statins lower plasmalemmal caveolin levels, thus decreasing caveolin-mediated eNOS inhibition [52].

\section{iNOS Inhibition}

Too little NO over the long term engenders cardiometabolic disorders. At the opposite extreme, the acute inflammatory induction of iNOS, as during sepsis, anaphylactic or cardiogenic shock or transplant organ rejection, drastically elevates NO levels. Excessive NO destroys mitochondrial function and is cytotoxic. It can evoke profound vasodilation, refractory hypotension, acute catecholamine-resistant cardiac pump failure and failure of multiple end-organs [22].

Such acute hemodynamic decompensation would be expected to benefit from an inhibition of NO overproduction. In contrast to the detrimental effects of nonselective NOS inhibition, selective iNOS inhibition may have therapeutic promise. In various animal studies, selective iNOS inhibition appears to attenuate sepsis-induced organ dysfunction and improve survival [53].

\section{Conclusion}

Vascular and metabolic health are interdependent. Anabolic metabolism requires not only nutrient intake but also vascular delivery of nutrients and anabolic hormones, like insulin, to target tissues.

Both the insulin receptor and NOS are expressed in the vascular endothelium, where they regulate vascular tone, as well as in skeletal and cardiac muscle, where they participate in metabolic processes. In fact, the insulin receptor and NOS are closely linked anatomically and functionally. In reciprocal fashion, insulin activates NOS, while $\mathrm{NO} / \mathrm{cGMP} / \mathrm{cGK}$ signaling enhances insulin sensitivity. Not surprisingly, preservation of normal NO signaling correlates with insulin-mediated glucose homeostasis.

In contrast, stress and inflammation are catabolic processes. Inflammatory processes prioritize nutrient utilization by insulin-independent immune organs at the expense of the needs of insulin-dependent tissues, such as the musculature. Inflammation engenders not only resistance to anabolic insulin actions but also vascular dysfunction with impaired nutrient delivery, in effect, the parallel disruption of metabolic-vascular insulin and NO signaling. This linkage between NO and insulin signaling is exemplified by murine insulin-receptor- or IRS-1knockout models, which develop endothelial dysfunction together with insulin resistance. It is also evident in murine eNOS-knockout models that acquire insulin resistance together with endothelial dysfunction. In practice, endothelial dysfunction compromises insulin sensitivity, insulin resistance worsens endothelial function, and the degree of endothelial dysfunction correlates with the severity of insulin resistance and contributes to its deterioration.

Any substrate of chronic stress and inflammation, even that associated with advancing age, will thus present with parallel manifestations of dysfunctional NO signal- 
ing and insulin resistance affecting many tissues, including the vasculature, the myocardium and the musculature. The ensuing vascular dysfunction and metabolic disturbances over time evolve into cardiometabolic diseases, as shown in table 2 .

The serious nature of the cardiometabolic diseases warrants preventive and therapeutic measures. Therapeutic lifestyle changes, such as calorie restriction, maintenance of optimal body weight, an anti-inflammatory diet rich in polyphenols, nitrate and nitroso-compounds and regular moderate physical training, are strategies that increase NO bioavailability and effectively prevent and/or reverse the systemic progression of cardiometabolic derangements.

In addition, effective prevention or intervention may require pharmacologic measures. Established NO donors are used in the treatment of angina, cardiomyopathy or pulmonary hypertension but have not been applied to in- sulin-resistant metabolic disease. However, the intricate $\mathrm{NO}$-insulin linkage provides a rationale for the future study of NO-based therapies for such disease. Potential therapies may target the release of small, physiologic NO amounts, or increases in the expression/activity of constitutional NOS, or upregulation of downstream NOcGMP signaling.

For the time being, a number of pharmacologic interventions, including statins, ACE inhibitors/ARBs and vasodilating $\beta$-blockers, do concomitantly enhance $\mathrm{NO}$ bioactivity and endothelial function coupled with an improvement in insulin sensitivity. Many are of proven benefit in improving cardiovascular prognosis, reducing macrovascular disease and mortality and lessening the risk of incident type $2 \mathrm{DM}$. Combination therapy with such agents, where indicated, may demonstrate not only additive beneficial effects but also positive synergisms.

\section{References}

$>_{1}$ Spier SA, Delp MD, Stallone JN, Dominguez JM 2nd, Muller-Delp JM: Exercise training enhances flow-induced vasodilation in skeletal muscle resistance arteries of aged rats: role of PGI2 and nitric oxide. Am J Physiol Heart Circ Physiol 2007;292:H3119-H3127.

-2 Clementi E, Nisoli E: Nitric oxide and mitochondrial biogenesis: a key to long-term regulation of cellular metabolism. Comp Biochem Physiol A Mol Integr Physiol 2005; 142 102-e110.

3 Marsh N, Marsh A: A short history of nitroglycerine and nitric oxide in pharmacology and physiology. Clin Exp Pharmacol Physiol 2000;27:313-319.

4 The Nobel Prize in Physiology or Medicine. Nobelprize.org. 2012. http://www.nobelprize.org/nobel_prizes/medicine/.

5 Ignarro LJ: Preface to this special journal issue on nitric oxide chemistry and biology. Arch Pharm Res 2009;32:1099-1101.

6 Kone BC, Kuncewicz T, Zhang W, Yu ZY: Protein interactions with nitric oxide synthases: controlling the right time, the right place, and the right amount of nitric oxide. Am J Physiol Renal Physiol 2003;285:F178F190.

7 Lundberg JO: Nitric oxide metabolites and cardiovascular disease. Markers, mediators, or both? J Am Coll Cardiol 2006;47:580-581.

-8 Miller MR, Megson IL: Recent developments in nitric oxide donor drugs. Br J Pharmacol 2007;151:305-321.
9 Munzel T, Feil R, Mulsch A, Lohmann SM, Hofmann F, Walter U: Physiology and pathophysiology of vascular signaling controlled by guanosine $3^{\prime}, 5^{\prime}$-cyclic monophosphatedependent protein kinase. Circulation 2003; 108:2172-2183.

10 Lima B, Forrester MT, Hess DT, Stamler JS S-Nitrosylation in cardiovascular signaling. Circ Res 2010;106:633-646.

-11 Parihar MS, Nazarewicz RR, Kincaid E, Bringold U, Ghafourifar P: Association of mitochondrial nitric oxide synthase activity with respiratory chain complex I. Biochem Biophys Res Commun 2008;1;366:23-28.

12 Gutierrez J, Ballinger SW, Darley-Usmar VM, Landar A: Free radicals, mitochondria, and oxidized lipids: the emerging role in signal transduction in vascular cells. Circ Res 2006;99:924-932.

13 Nisoli E, Carruba MO: Nitric oxide and mitochondrial biogenesis. J Cell Sci 2006;119: 2855-2862.

14 Dedkova EN, Blatter LA: Modulation of mitochondrial $\mathrm{Ca} 2+$ by nitric oxide in cultured bovine vascular endothelial cells. Am J Physiol Cell Physiol 2005;289:C836-C845.

15 Erusalimsky JD, Moncada S: Nitric oxide and mitochondrial signaling: from physiology to pathophysiology. Arterioscler Thromb Vasc Biol 2007;27:2524-2531.

16 Duchen MR: Roles of mitochondria in health and disease. Diabetes 2004;53(suppl 1):S96S102.

17 Guarente L: Mitochondria - a nexus for aging, calorie restriction, and sirtuins? Cell 2008;132:171-176.
18 Gutierrez J, Ballinger SW, Darley-Usmar VM, Landar A: Free radicals, mitochondria, and oxidized lipids: the emerging role in signal transduction in vascular cells. Circ Res 2006;99:924-932.

19 Zenebe WJ, Nazarewicz RR, Parihar MS, Ghafourifar P: Hypoxia/reoxygenation of isolated rat heart mitochondria causes cytochrome $\mathrm{c}$ release and oxidative stress; evidence for involvement of mitochondrial nitric oxide synthase. J Mol Cell Cardiol 2007; 43:411-419.

20 Mitsuishi M, Miyashita K, Itoh H: cGMP rescues mitochondrial dysfunction induced by glucose and insulin in myocytes. Biochem Biophys Res Commun 2008;21;367:840-845.

21 Rakhit RD, Mojet MH, Marber MS, Duchen MR: Mitochondria as targets for nitric oxide-induced protection during simulated ischemia and reoxygenation in isolated neonatal cardiomyocytes. Circulation 2001; 103: 2617-2623.

22 Crouser ED: Mitochondrial dysfunction in septic shock and multiple organ dysfunction syndrome. Mitochondrion 2004;4:729-741.

23 Kingwell BA: Nitric oxide-mediated metabolic regulation during exercise: effects of training in health and cardiovascular disease. FASEB J 2000;14:1685-1696.

24 Begum N, Sandu OA, Ito M, Lohmann SM, Smolenski A: Active Rho kinase (ROK-alpha) associates with insulin receptor substrate- 1 and inhibits insulin signaling in vascular smooth muscle cells. J Biol Chem 2002; 277:6214-6222. 
-25 Higaki Y, Hirshman MF, Fujii N, Goodyear LJ: Nitric oxide increases glucose uptake through a mechanism that is distinct from the insulin and contraction pathways in rat skeletal muscle. Diabetes 2001;50:241-247.

26 Jobgen WS, Fried SK, Fu WJ, Meininger CJ, Wu G: Regulatory role for the arginine-nitric oxide pathway in metabolism of energy substrates. J Nutr Biochem 2006;17:571-588.

-27 Finkel MS, Oddis CV, Jacob TD, Watkins SC, Hattler BG, Simmons RL: Negative inotropic effects of cytokines on the heart mediated by nitric oxide. Science 1992;257:387-389.

$\checkmark 28$ Hayden MR, Tyagi SC: Is type 2 diabetes mellitus a vascular disease (atheroscleropathy) with hyperglycemia a late manifestation? The role of NOS, NO, and redox stress. Cardiovasc Diabetol 2003;2:2.

-29 Aicher A, Heeschen C, Mildner-Rihm C, Urbich C, Ihling C, Technau-Ihling K, Zeiher AM, Dimmeler S: Essential role of endothelial nitric oxide synthase for mobilization of stem and progenitor cells. Nat Med 2003;9: 1370-1376.

30 Levonen AL, Patel RP, Brookes P, Go YM, Jo H, Parthasarathy S, Anderson PG, DarleyUsmar VM: Mechanisms of cell signaling by nitric oxide and peroxynitrite: from mitochondria to MAP kinases. Antioxid Redox Signal 2001;3:215-229.

-31 Förstermann U: Nitric oxide and oxidative stress in vascular disease. Pflügers Arch 2010;459:923-939.

- 32 Kim F, Pham M, Maloney E, Rizzo NO, Morton GJ, Wisse BE, Kirk EA, Chait A, Schwartz MW: Vascular inflammation, insulin resistance, and reduced nitric oxide production precede the onset of peripheral insulin resistance. Arterioscler Thromb Vasc Biol 2008; 28:1982-1988.

-33 Cooke JP: Does ADMA cause endothelial dysfunction? Arterioscler Thromb Vasc Biol 2000;20:2032-2037.
34 Colombo MG, Andreassi MG, Paradossi U, Botto N, Manfredi S, Masetti S, Rossi G, Clerico A, Biagini A: Evidence for association of a common variant of the endothelial nitric oxide synthase gene (Glu298 $\rightarrow$ Asp polymorphism) to the presence, extent, and severity of coronary artery disease. Heart 2002,87:525-528.

35 Romero MJ, Platt DH, Tawfik HE, Labazi M, El-Remessy AB, Bartoli M, Caldwell RB, Caldwell RW: Diabetes-induced coronary vascular dysfunction involves increased arginase activity. Circ Res 2008;102:95-102.

36 Kim F, Gallis B, Corson MA: TNF-alpha inhibits flow and insulin signaling leading to NO production in aortic endothelial cells. Am J Physiol Cell Physiol 2001;280:C1057C1065.

37 Dandona P, Aljada A, Chaudhuri A, Mohanty P, Garg R: Metabolic syndrome. A comprehensive perspective based on interactions between obesity, diabetes, and inflammation. Circulation 2005;111:1448-1454.

- 38 Hsueh WA, Quinones MJ: Role of endothelial dysfunction in insulin resistance. Am J Cardiol 2003;92(suppl):10J-17J.

-39 Kingwell BA: Nitric oxide-mediated metabolic regulation during exercise: effects of training in health and cardiovascular disease. FASEB J 2000;14:1685-1696.

40 Davies PF, Civelek M, Fang Y, Guerraty MA, Passerini AG: Endothelial heterogeneity associated with regional athero-susceptibility and adaptation to disturbed blood flow in vivo. Semin Thromb Hemost 2010;36:265-275.

-41 Suvorava T, Lauer N, Kojda G: Physical inactivity causes endothelial dysfunction in healthy young mice. J Am Coll Cardiol 2004; 44:1320-1327.

42 Libby P: Inflammation in atherosclerosis. Nature 2002;420:868-874.

43 Widlansky ME, Gokce N, Keaney JJ, Vita JA: The clinical implications of endothelial dysfunction. J Am Coll Cardiol 2003;42:11491160.

44 Duplain H, Burcelin R, Sartori C, Cook S, Egli M, Lepori M, Vollenweider P, Pedrazzini T, Nicod P, Thorens B, Scherrer U: Insulin resistance, hyperlipidemia, and hypertension in mice lacking endothelial nitric oxide synthase. Circulation 2001;104:342-345.
45 Rizzo NO, Maloney E, Pham M, Luttrell I, Wessells H, Tateya S, Daum G, Handa P, Schwartz MW, Kim F: Reduced NO-cGMP signaling contributes to vascular inflammation and insulin resistance induced by highfat feeding. Arterioscler Thromb Vasc Biol 2010;30:758-765.

46 Zhang Y, Janssens SP, Wingler K, Schmidt $\mathrm{HH}$, Moens AL: Modulating endothelial nitric oxide synthase: a new cardiovascular therapeutic strategy. Am J Physiol Heart Circ Physiol 2011;301:H634-H646.

47 Nisoli E, Tonello C, Cardile A, Cozzi V, Bracale R, Tedesco L, Falcone S, Valerio A, Cantoni O, Clementi E, Moncada S, Carruba MO: Calorie restriction promotes mitochondrial biogenesis by inducing the expression of eNOS. Science 2005;310:314-317.

48 Jankowski P, Safar ME, Benetos A: Pleiotropic effects of drugs inhibiting the renin-angiotensin-aldosterone system. Curr Pharm Des 2009; 15:571-584.

- 49 Javed U, Deedwania PC: Angiotensin receptor blockers: novel role in high-risk patients. Cardiol Clin 2008;26:507-526.

50 Kamp O, Metra M, Bugatti S, Bettari L, Dei Cas A, Petrini N, Dei Cas L: Nebivolol: haemodynamic effects and clinical significance of combined beta-blockade and nitric oxide release. Drugs 2010;70:41-56.

51 Schwartz BG, Levine LA, Comstock G, Stecher VJ, Kloner RA: Cardiac uses of phosphodiesterase-5 inhibitors. J Am Coll Cardiol 2012;59:9-15.

52 Liao JK, Laufs U: Pleiotropic effects of statins. Annu Rev Pharmacol Toxicol 2005; 45:89-118

53 Heemskerk S, Masereeuw R, Russel FG, Pickkers P: Selective iNOS inhibition for the treatment of sepsis-induced acute kidney injury. Nat Rev Nephrol 2009;5:629-640. 